\title{
Comportamento do boto-cinza, Sotalia guianensis (Van Bénédén) (Cetacea, Delphinidae) na presença de barcos de turismo na Praia de Pipa, Rio Grande do Norte, Brasil
}

\author{
Érico Santos-Jr ${ }^{1}$; Kelly C. Pansard ${ }^{1}$; Maria E. Yamamoto ${ }^{1} \&$ Sathyabama Chellappa ${ }^{2}$ \\ 1 Programa de Pós-Graduação em Psicobiologia, Departamento de Fisiologia, Centro de Biociências, Universidade Federal do \\ Rio Grande do Norte. Rua Senador Salgado Filho 3000, 59072-970 Natal, Rio Grande do Norte, Brasil. \\ E-mail: esanior@hotmail.com; emilia.yamamoto@uol.com.br \\ 2 Programa de Pós-Graduação em Bioecologia Aquática, Departamento de Oceanografia e Limnologia, Centro de \\ Biociências, Universidade Federal do Rio Grande do Norte. Praia de Mãe Luíza, 59014-100 Natal, Rio Grande do Norte, \\ Brasil.E-mail: bama@dol.ufrn.br
}

\begin{abstract}
Behavior of estuarine dolphin, Sotalia guianensis (Van Bénédén) (Cetacea, Delphinidae) in the presence of tourist boats in Pipa Beach, Rio Grande do Norte, Brazil. The ever-increasing tourist industry has extended to the aquatic environments, thus making the aquatic mammals susceptible to interactions with humans. Dolphin watching tourism has developed to a great extent in the Pipa Beach, Rio Grande do Norte, consequently the number of dolphin-watching boats operating in this area has increased. In the present study the behavioral alterations of Sotalia guianensis (Van Bénédén, 1864) caused by the presence of these boats were verified. Behavioral registers of $S$. guianensis were compiled from a fixed point on the cliffs close to the study area. The observations were effected when there were no boats, in the presence of boats and after the departure of boats. The results show that type of approach by the dolphin watching boats had a major influence on the behavior of the dolphins, especially so in the groups with calves. The short term impacts were rather discrete, however, new methods should be applied in order to verify the long term impacts on the dolphins.

KEY WORDS. Aquatic mammals; behavior; estuarine dolphin; watching tourism.
\end{abstract}

RESUMO. A crescente indústria do turismo tem se estendido ao ambiente aquático, deste modo tornando os mamíferos aquáticos suscetíveis a interações com humanos. O turismo de observação de golfinhos na praia de Pipa litoral sul do Rio Grande do Norte, tem se desenvolvido aumentando o número de barcos de observação de golfinhos operando nesta área. No presente estudo alterações comportamentais do Sotalia guianensis (Van Bénédén, 1864) causado pela presença destes barcos foi verificada. Registros comportamentais de $S$. guianensis foram compilados de um ponto fixo do alto de uma falésia próxima à área de estudo. As observações foram realizadas quando não havia barcos, na presença de barcos e após a saída dos barcos. Os resultados mostraram que o tipo de aproximação praticado pelos barcos de observação de golfinhos tinha maior influência no comportamento dos animais, especialmente em grupos com filhotes. Os impactos de curto prazo foram mais discretos, no entanto, novos métodos devem ser aplicados no sentido de verificar impactos de longo prazo no comportamento dos golfinhos.

PALAVRAS-CHAVE. Boto-cinza; comportamento; mamíferos aquáticos; turismo de observação.

Entre as mais variadas modalidades de ecoturismo, um deles, voltado para avistagem de mamíferos aquáticos, principalmente golfinhos e baleias, têm mostrado grande potencial de crescimento. O Comitê Científico da International Whaling Comission (IWC) considerando o desenvolvimento acelerado do turismo de observação de baleias e golfinhos e seu inegável benefício econômico (Williams et al. 2002), desenvolveu dire- trizes para sua regulação estabelecendo algumas prioridades, de modo que ao mesmo tempo tem encorajado o adiantado desenvolvimento do turismo de observação, bem como o uso sustentável desses recursos.

Na Escócia, no estuário de Morey Firth, as interações dos barcos de turismo com o golfinho nariz-de-garrafa, Tursiops truncatus (Montagu, 1821), causaram uma diminuição no com- 
portamento de superfície destes animais (JANIK \& THOMPSON 1996). Em belugas Delphinapterus leucas (Pallas, 1776), no estuário de St. Lawrence, Canadá, as respostas foram seqüências repetidas de vocalizações (LÈsAGE et al. 1999). Um outro exemplo, novamente com o golfinho nariz-de-garrafa, sugere que o aumento do tráfego de barcos para avistagem de cetáceos, além de embarcações recreativas e comerciais na Florida provocou alterações na seleção de habitat para forrageio, levando os indivíduos a buscar por águas mais profundas em canais dragados ou por águas mais rasas (Allen \& READ 2000).

Na praia de Pipa, localizada no Município de Tibau do Sul no Rio Grande do Norte, o boto-cinza, Sotalia guianensis (Van Bénédén, 1864) é avistado diariamente (ARAújo et al. 2001). As enseadas das praias do Madeiro e do Curral são utilizadas pelos animais para obtenção de recursos alimentares, para reprodução e socialização (SPINELl et al. 2002). Simultaneamente dentro dessas enseadas ocorrem diariamente passeios de barcos de turismo com objetivo de avistagem desses animais, em números crescentes.

Estudos semelhantes a este, com a mesma espécie ainda são escassos foram realizados em diferentes regiões com diferentes dinâmicas costeiras (GEISE et al. 1999, SANTOS et al. 2000). No entanto, pela ocupação de habitats costeiros, esta espécie é especialmente suscetível a tornar-se o foco deste tipo de atividade de observação de golfinhos. Este estudo investigou as respostas comportamentais de boto-cinza ao turismo de avistagem na praia de Pipa, Rio Grande do Norte.

\section{MATERIAL E MÉTODOS}

A praia do Curral no distrito de Pipa $6^{\circ} 13^{\prime} 36,7^{\prime \prime} \mathrm{S}, 35^{\circ} 3^{\prime}$ 36,7"W no Município de Tibau do Sul, a $85 \mathrm{~km}$ de Natal, no Rio Grande do Norte, é caracterizada por apresentar formações rochosas de arenito e enseada fechada com falésias que chegam a alcançar até $30 \mathrm{~m}$ de altura. É bastante influenciada pelas variações de marés e apresenta uma topografia subaquática com declive suave e extenso, constituído por fundo arenoso e rochoso com profundidade de até 7,6 m.

As observações foram efetuadas de um ponto fixo no alto da falésia da praia do Curral utilizando-se planilhas de dados e gravador de voz para o registro sistemático através da técnica de amostragem contínua (Altmann 1974). Dois observadores treinados utilizavam binóculos para facilitar a observação e identificação das atividades comportamentais e localização espacial dos animais, e registraram a presença de animais e de barcos de turismo dentro da área de estudo em intervalos regulares de cinco minutos.

Para tornar mais fácil a visualização dos golfinhos na área de estudo e facilitar sua localização na enseada, esta foi dividida em quatro quadrantes imaginários (Fig. 1).

Os grupos avistados no quadrante Q4 eram apenas acompanhados visualmente e registrava-se a presença e o número de indivíduos nas planilhas. Quando estes mesmos grupos se deslocavam para um dos outros três quadrantes passavam a ser um

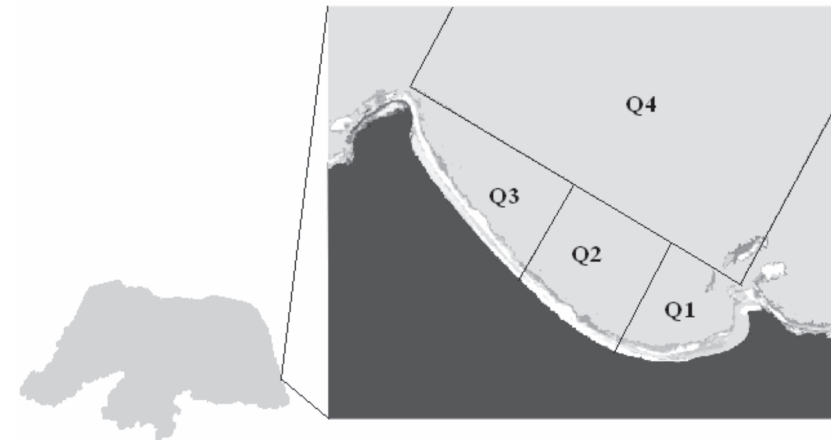

Figura 1. Enseada da praia do Curral, Tibau do Sul, Rio Grande do Norte, divisão da área de estudo em quadrantes através de linhas imaginárias para facilitar a visualização e a localização dos golfinhos na enseada.

grupo focal. Esta condição de acompanhamento visual somente foi realizada quando não havia outros indivíduos dentro dos quadrantes Q1, Q2 ou Q3. Quando não era avistado qualquer indivíduo dentro da enseada, em intervalos de 10 minutos, era feita uma varredura em todos os quadrantes de observação principalmente no Q4 para verificar eventuais grupos que pudessem se deslocar para os quadrantes Q1, Q2 e/ou Q3. Quando um grupo entrava ou era avistado nos quadrantes 1, 2 ou 3 anotavase seu tamanho e composição em termos de faixa etária.

Foi considerado como um grupo aquele em que os indivíduos presentes na enseada nadavam próximos uns dos outros, com atividades coordenadas, mas não necessariamente deslocando-se na mesma direção (MANN 2000). A identificação de faixa etária foi feita de acordo com Spinelu et al. (2002), pela comparação do tamanho entre os animais do grupo observado, considerando filhotes e adultos. Para a classificação dos indivíduos nessas faixas etárias era necessária a concordância entre os observadores quanto ao tamanho aproximado do animal.

Durante todo o período do estudo, foram realizadas observações em quatro dias/mês, de janeiro de 2000 a julho de 2002. O esforço amostral diário foi de 12 horas no ano de 2000 passando para 6 horas em 2001 e 2002, iniciando-se as observações entre as primeiras horas do dia claro ou no final do turno da manhã. O esforço efetivo que incluía os períodos de antes, durante e após as interações de golfinhos com barcos de turismo foi de $146 \mathrm{~h}$.

Os dados foram coletados em três períodos diferentes: (1) um período de 20 minutos sem a presença de barcos na enseada durante o qual foram anotados os comportamentos dos golfinhos; (2) um período em que os golfinhos foram avistados na enseada na presença de embarcações. O período se iniciava quando barcos e golfinhos se encontravam no mesmo quadrante, o que em geral ocorria pela movimentação dos barcos em direção aos animais. Este período foi encerrado quando os barcos deixavam a enseada, o que variou de 5 a 80 minutos. Durante este período podiam ocorrer interações entre os animais e os barcos. 
Estas interações poderiam ser com um único barco, dois barcos simultaneamente ou dois barcos entrando em seqüência na enseada; (3) um período de pós-exposição, que consistia em um período de 20 minutos imediatamente após a saída dos barcos da enseada no qual se encontravam os animais.

Durante os três períodos foram registrados todos os comportamentos apresentados pelos animais e agrupados nas seguintes categorias: alimentação ou forrageio (A) - animais nadando em velocidade com a região ventral próxima a superfície podendo ou não ocorrer giros, sempre sendo finalizado com bote expondo o corpo total ou parcialmente na a superfície da água; socialização (SO) - animais nadando na superfície com contatos físicos em pequenos grupos podendo ocorrer comportamentos aéreos como saltos, periscópios e batidas com o corpo; deslocamentos (D) - quando os animais movimentavam-se de uma área a outra mantendo direção fixa, alternando mergulhos com rápidas subidas à superfície para respirar; e comportamentos aéreos (CA) - toda a atividade em que os animais expunham-se total ou parcialmente acima da superfície da água, exceto nos deslocamentos os quais os animais subiam somente para respirar e no bote usado na captura de presas.

Durante o período de coleta de dados, três embarcações de turismo de modelo e características diferentes, bem como, os modos de aproximação aos golfinhos, freqüentaram a área do estudo. O tipo de aproximação combinou velocidade e direção das embarcações da seguinte forma: rápida e direcional quando o barco avançou de forma acelerada e seguiu em direção ao grupo de golfinhos; lenta e direcional - o barco avançou de forma lenta e seguiu em direção ao grupo de golfinhos. Para cada episódio de interação foi registrada, a embarcação ou embarcações presentes, a seqüência em que entravam na enseada e o tipo de aproximação utilizada (Tab. I).

\section{RESULTADOS}

Durante todo o período da pesquisa foi registrado um número total de 120 episódios dentre os quais 54 com a presença de um único barco, 39 com dois barcos e 27 episódios com os barcos entrando em seqüência na enseada da praia do Curral. Em todos os dias de observação ocorreram encontros entre barcos e golfinhos exceto no mês de janeiro de 2001 quando foi verificada presença de golfinhos, mas não ocorreram episódios de interação com barcos. O número de visitas para cada barco variou de uma a três vezes ao longo do período diário de observação. O tempo médio de duração de todas as categorias de interação foi de 34,8 minutos sendo 25,8 minutos para barcos isolados, 31,7 minutos para mais de um barco e 53,6 minutos para barcos entrando em seqüência na enseada da praia do Curral.

\section{Tipos de abordagens dos barcos}

A avaliação das alterações no comportamento em função do tipo de aproximação foi feita apenas quando havia um único barco na enseada. Não foram encontradas alterações na alimentação $\left(\mathrm{H}_{(2,54)}=2,4195 ; \mathrm{p}=0,2983\right)$, comportamento aéreo $\left(\mathrm{H}_{(2,54)}=1,3532 ; \mathrm{p}=0,5084\right)$ ou socialização $\left(\mathrm{H}_{(2,54)}=2,5016\right.$; $\mathrm{p}=0,2863)$ na comparação das situações controle, experimental e pós-exposição utilizando o teste de Kruskal-Wallis. Entretanto, foram encontradas diferenças significativas para a categoria deslocamento entre as três situações $\left(\mathrm{H}_{(2,54)}=7,7089 ; \mathrm{p}=0,0212\right)$.

Separando a amostra de acordo com os diferentes tipos de barcos e aproximação (Tab. I), para aquele com aproximação mais rápida e direcional não foi encontrada qualquer diferença no deslocamento $\left(\mathrm{H}_{(2,30)}=2,3957 ; \mathrm{p}=0,3019\right)$, enquanto, para o barco de aproximação lenta e direcional foram encontradas diferenças $\left(\mathrm{H}_{(2,24)}=7,1399 ; \mathrm{p}=0,0282\right)$. O teste $\mathrm{U}$ de Mann-Whitney, então foi utilizado, para comparação as situações, duas a duas. A média de deslocamento na situação experimental, tanto para o barco de aproximação lenta e direcional $(2,73 \pm 3,57)$ quanto para o barco de aproximação rápida e direcional $(0,69 \pm 1,90)$, mostraram valores acima das situações controle e pós-exposição (Fig. 2).

Aplicado o coeficiente de correlação não-paramétrica de Spearman para verificar se existiam correlações significativas, na condição de barco isolado, na comparação das categorias comportamentais de alimentação (A), socialização (SO) e atividades aéreas (CA), em relação ao deslocamento (D) praticado pelo $S$. guianensis, não houve qualquer correlação entre as categorias.

\section{Respostas comportamentais de grupos com filhotes}

Foram avaliados as alterações comportamentais de grupos de golfinhos com filhotes em comparação com aqueles sem filhotes, nas interações com os três tipos de condições (barco isolado, ambos os barcos e barcos entrando em seqüência na enseada). A amostra foi dividida em grupos sem filhotes, grupos com um único filhote e grupos com mais de um filhote. A condição de barco isolado mostrou diferenças significativas $\left(\mathrm{H}_{(2,54)}=9,7541 ; \mathrm{p}=0,0076\right)$ para o comportamento aéreo entre as três composições de grupos e comparado os três tipos de composição dois a dois. O teste de Mann-Whitney revelou que os grupos sem filhotes mostraram significativamente mais comportamentos aéreos do que os outros dois tipos de grupos na presença de barcos isolados (Fig. 3).

Tabela I. Características dos barcos e forma de aproximação aos grupos de golfinhos.

\begin{tabular}{cccccc}
\hline Barco & Modelo & Comprimento $(\mathrm{m})$ & Motor & Capacidade (pessoas) & Aproximação \\
\hline Malembar I & Escuna & 13,7 & MWM 6 cc & 65 & Rápida e direcional \\
Aventureiro & Saveiro & 15,0 & MWM 6 cc & 60 & Lenta e direcional \\
Malembar II & Escuna & 17,0 & MWM 8 cc & 75 & Rápida e direcional \\
\hline
\end{tabular}

Revista Brasileira de Zoologia 23 (3): 661-666, setembro, 2006 

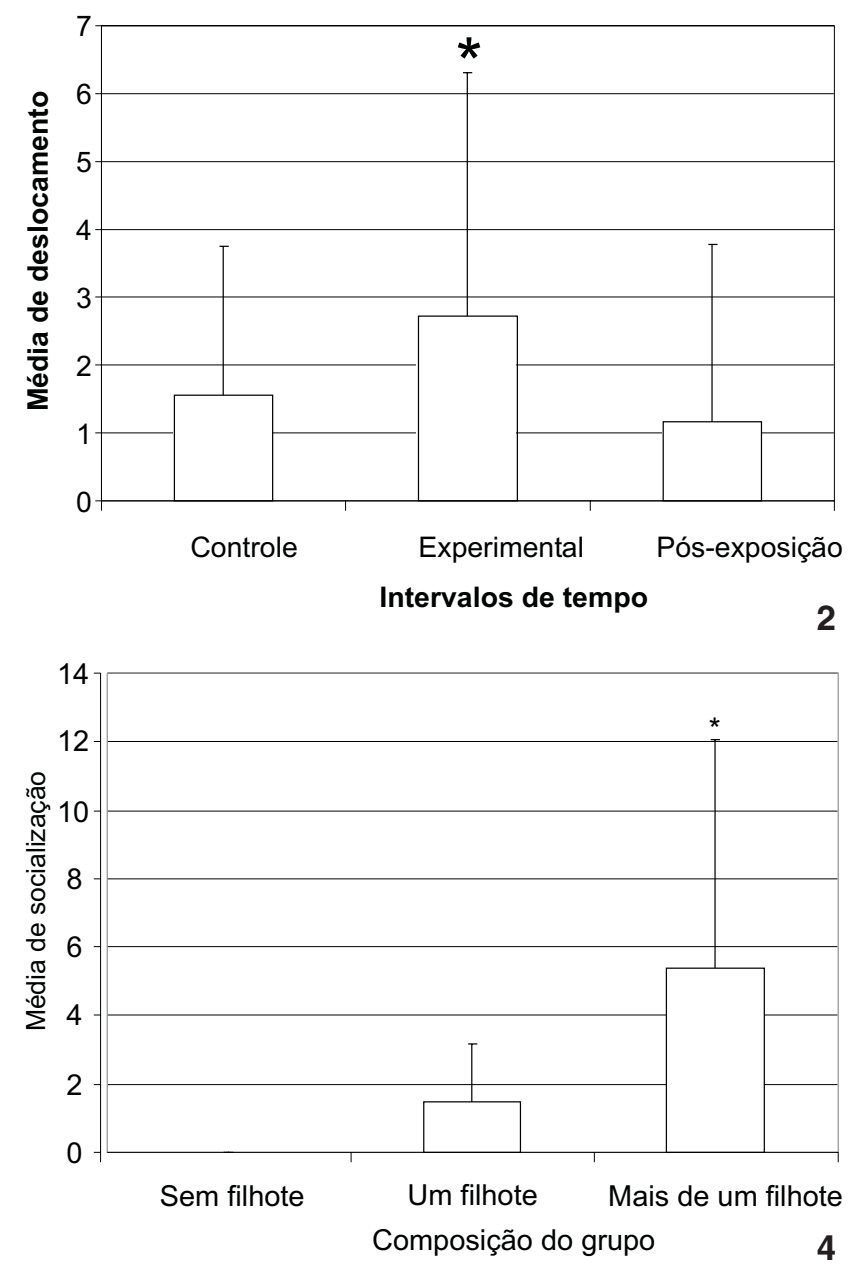
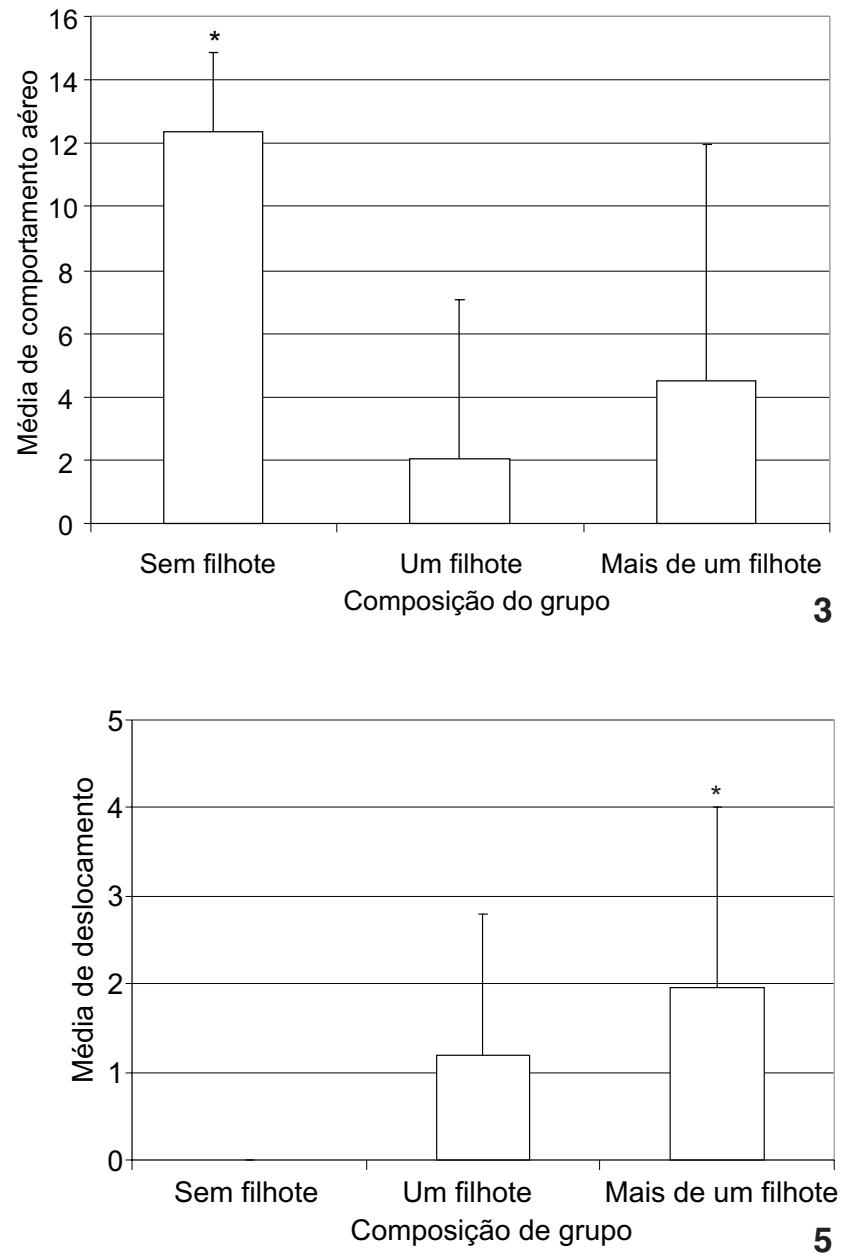

Figuras 2-5. Comparação das médias das freqüências de deslocamento ( \pm EP), para as situações de controle, experimental e pós-exposição, durante interações com embarcação de com aproximação lenta e direcional; (3) média de comportamentos aéreos ( \pm EP) para cada um dos tipos de composição de grupo nas interações com barcos isolados; (4) média de socialização ( \pm EP) para cada um dos tipos de composição de grupo nas interações com barco isolado; (5) Média de deslocamento ( \pm EP) para cada um dos tipos de composição de grupo nas interações com barco isolado. $\left({ }^{*}\right)$ Composição de grupo com diferenças significativas, $p<0,05$ pelo teste $U$ de Mann-Whitney.

O comportamento alimentar não mostrou diferença significativa $\left(\mathrm{H}_{(2,54)}=3,9031 ; \mathrm{p}=0,1421\right)$ entre os tipos de composição de grupo testados. No entanto, diferenças foram percebidas para socialização $\left(\mathrm{H}_{(2,54)}=5,9469 ; \mathrm{p}=0,0500\right)$ e deslocamento $\left(\mathrm{H}_{(2,54)}=7,0033 ; \mathrm{p}=0,0301\right)$, que foram comparados dois a dois (Figs 4 e 5).

Para a condição em que os dois barcos interagiram com os grupos de golfinhos, nenhuma categoria comportamental: comportamento aéreo $\left(\mathrm{H}_{(2,39)}=4,5510 ; \mathrm{p}=0,1028\right)$, alimentação $\left(\mathrm{H}_{(2,39)}=0,9311 ; \mathrm{p}=0,6278\right)$, socialização $\left(\mathrm{H}_{(2,39)}=1,3559\right.$; $\mathrm{p}=0,5077)$ ou deslocamento $\left(\mathrm{H}_{(2,39)}=2,8306 ; \mathrm{p}=0,2429\right)$ apresentou quaisquer diferenças significativas entre os três tipos de composição de grupo.

Para os episódios de interações que ocorreram entre os gru- pos de golfinhos e barcos que entraram em seqüência na enseada da praia do Curral, também não foram encontradas quaisquer diferenças significativas para as categorias comportamentais testadas: comportamento aéreo $\left(\mathrm{H}_{(2,27)}=0,0313 ; \mathrm{p}=0,8596\right)$, alimentação $\left(H_{(2,27)}=0,0638 ; \mathrm{p}=0,8006\right)$, socialização $\left(\mathrm{H}_{(2,27)}=0,8491\right.$; $\mathrm{p}=0,3568)$ ou deslocamento $\left(\mathrm{H}_{(2,27)}=0,9594 ; \mathrm{p}=0,3273\right)$.

\section{DISCUSSÃO}

Os modelos de barcos que interagem com cetáceos em regiões onde há o turismo para avistagem destes animais variam amplamente. Suas características estruturais, mecânicas e tipo de abordagem podem, em maior ou menor grau, causar alterações comportamentais nos indivíduos alvo em curto e longo prazo (Watkins 1986, Bedjer et al. 1999, Constantine 2001, NowaceK 
et al. 2001, WiLLIAMs et al. 2002).

Os resultados sugerem que a forma de aproximação praticada pelos diferentes barcos pode, na praia de Pipa, não estar relacionada a alterações do deslocamento dos grupos de golfinhos na enseada. No entanto, os barcos possuem características físicas e mecânicas distintas que podem ser percebidas pelo boto-cinza, como, por exemplo, ruídos sub-aquáticos do funcionamento dos motores, cavitação, aceleração súbita e partida de motores, além de ruídos aéreos provocados pelos turistas e aparelhos sonoros a bordo das embarcações, e até mesmo, o tamanho e modelo da embarcação. BEDJER et al. (1999) consideraram uma situação como de impacto potencial quando embarcações estavam em qualquer local da área de estudo, enquanto NowACEK et al. (2001) consideraram impactante, qualquer barco a uma distância de menos de $100 \mathrm{~m}$ dos golfinhos.

A conduta utilizada pelos operadores dos barcos durante interações com o boto-cinza pode induzir alterações sutis nas categorias comportamentais praticadas pelos golfinhos. Isto foi verificado apenas para o deslocamento, e uma possível correlação entre ele e outros comportamentos não foi encontrada. Efeitos no padrão de movimentos são importantes para reconhecer que não só o afastamento, mas também reações aparentemente positivas, como aproximações, poderiam ter efeitos negativos em longo prazo se, por exemplo, eles influenciarem o tempo gasto na alimentação (JANIK \& THOMPSON 1996).

Os comportamentos apresentados por $S$. guianensis na praia de Pipa são caracterizados nas categorias descritas na metodologia e estão relacionados, cada um deles, com um contexto específico. O comportamento aéreo foi mais evidente quando não foi observada presença de filhotes. Isso pode estar relacionado ao comportamento de proteção dos indivíduos mais jovens pelos adultos em grupos com filhotes que evitam a interação ou saem da enseada, enquanto grupos somente com adultos podem interagir mais tempo nesta condição. Durante interações com barcos de turismo, fêmeas de baleia franca do sul Eubalaena autralis (Desmoulins, 1822), acompanhada de filhotes, evitaram freqüentemente barcos de turismo para avistagem de cetáceos (Rivarola et al. 2001).

As atividades na superfície como saltos, periscópios e batidas de nadadeira caudal estão relacionados à socialização, cópula e presença de embarcações. Embora possíveis funções de comportamentos aéreos desempenhados pelos cetáceos permanecem desconhecidas, NorRIs et al. (1994) sugeriram que alguns deles podem sinalizar situação de perigo ou representar ameaça, o que parece apropriado para a situação de aproximação dos barcos. Por outro lado, este tipo de comportamento põe o animal em evidência, expondo o grupo, o que talvez seja evitado quando há filhotes. Também é importante perceber que alterações nos comportamentos aéreos nas outras situações (com mais de um barco e barcos entrando em seqüência na enseada) não foram estatisticamente diferentes para as composições de grupos estudadas. Respostas diferenciadas entre as situações podem ser devidas aos animais não perceberem a presença do barco de turismo de uma única maneira.
Era esperado que os grupos com filhotes mostrassem mais alterações comportamentais durante as interações, o que foi de fato observado. Em todas as condições de interação a média de comportamentos indicativos de afastamento foi maior do que aqueles indicativos de aproximação. Isto foi mais claro na condição barco isolado sugerindo que o padrão de movimento pode ter relação com a baixa freqüência dos comportamentos aéreos apresentados pelos grupos com filhotes em sua composição. Reações de afastamento durante a interação com barcos pode ser interpretado como um comportamento de defesa, na forma de evitação, buscando proteção aos animais mais jovens através do deslocamento para fora da enseada da praia do Curral. Desta forma o afastamento diminui o tempo de interação, conseqüentemente reduzindo a freqüência de atividades aéreas para grupos com filhotes na composição. Por outro lado, grupos só com animais adultos podem interagir mais tempo com o barco.

Redução na atividade de alimentação foi percebida através da análise qualitativa dos dados. Atividades de forrageio, por exemplo, perseguição e captura de presas foram observadas mesmo com a presença das embarcações, mas somente nas partes mais rasas da enseada ou em áreas diferentes daquelas em que se encontravam os barcos. Tentativamente é possível supor que a presença dos barcos perturbe esta atividade específica dos animais ao provocar sua movimentação. Em favor desta conclusão, observamos maior afastamento do que aproximação aos barcos durante as condições de interações. Allen \& READ (2000) mencionam que uso de habitats de forrageio primário por T. truncatus diminuiu durante períodos de alta densidade de barcos. Os autores sugerem que os golfinhos poderiam mudar suas preferências de uso dos habitats diretamente para evitar áreas de alto tráfego de barcos ou indiretamente em resposta à reação das presas ao tráfego de barcos.

SPINelLi et al. (2002) sugerem que adultos de S. guianensis somente participem de brincadeiras em que aspectos sociais são mais evidentes do que brincadeiras que envolvam atividades locomotoras. Portanto, é de se esperar que em grupos com maior número de filhotes o aumento da socialização se deva ao aumento de brincadeiras locomotoras e não a outros comportamentos de interação.

\section{AGRADECIMENTOS}

À Coordenação de Aperfeiçoamento de Pessoal de Nível Superior (CAPES) e ao Conselho Nacional de Desenvolvimento Científico e Tecnológico (CNPq), ao Programa de Pós-Graduação em Psicobiologia da Universidade Federal do Rio Grande do Norte, ao Santuário Ecológico de Pipa e ao Projeto Pequenos Cetáceos.

\section{REFERÊNCIAS BIBLIOGRÁFICAS}

Allen, M.C. \& A.J. Read. 2000. Habitat selection of foraging bottlenose dolphins in relation to boat density near Clearwater, Florida. Marine Mammal Science, Lawrence, 16 (4): 815-824.

Revista Brasileira de Zoologia 23 (3): 661-666, setembro, 2006 
Altmann, J. 1974. Observational study of behavior: Sampling methods. Behavior, Netherlands, 49: 227-265.

Araujo, J.P.; J.Z.O. Passavante \& A.S. Souto. 2001. Behavior of the esturine dolphin, Sotalia guianensis, at Dolphin Bay Pipa - Rio Grande do Norte. Tropical Oceanography, Recife, 29 (2): 13-23.

Bedjer, L.; S.M. Dawson \& J.A. Harraway. 1999. Responses by Hector's dolphins toa boats and swimmers in Porpoise Bay, New Zeland. Marine Mammal Science, Lawrence, 15 (3): 738-750.

CONSTANTINE, R. 2001. Increased avoidance of swimmers by wild bottlenose dolphins (Tursiops truncatus) due to long-term exposure to swim-with-dolphin tourism. Marine Mammal Science, Lawrence, 17 (4): 689-702.

Geise, L.; N. Gomes \& R. Cerqueira. 1999. Behaviour, habitat use and population size of Sotalia fluviatilis (Gervais, 1853) (Cetacea, Delphinidae) in the Cananéia estuary region, São Paulo, Brazil. Revista Brasileira Biologia, Curitiba, 59 (2): 183-194.

JANIK, V.M. \& P.M. ThOMPson. 1996. Changes in surfacing patterns of bottlenose dolphins in response to boat traffic. Marine Mammal Science, Lawrence, 12 (3): 597-602.

Lesage, V.; C. Barrette; M.C.S. Kingsley \& B. SJare. 1999. The effect of vessel noise on the vocal behavior of belugas in the St. Lawrence river estuary, Canada. Marine Mammal Science, Lawrence, 15 (1): 65-84.

Mann, J. 2000. Unraveling the dynamics of social life, p. 45-64. In: J. Mann; R.C. Connor; P.L. Tyack \& H. Whitehead (Eds). Cetacean society - field studies of dolphin and whales. Chicago, University Chicago Press, 448p.

Recebido em 15.III.2005; aceito em 15.VIII.2006.
NorRIS, K.S.; B. WÜrsig \& R.S. Wells. 1994. Aerial behavior Slaps, p. 103-121. In: K.S. NorRIs; B. WürSIG; R.S. Wells \& M. WÜRSIG (Eds). The Hawaiian spinner dolphin. California, University of California Press, 408p.

NowACEK, S.M.; R.S. Wells \& A.R. Solow. 2001. Shot-term effects of boat traffic on bottlenose dolphins, Tursiops truncatus, in Sarasota Bay, Florida. Marine Mammal Science, Lawrence, 17 (4): 673-688.

Rivarola, M.; C. Campagna \& A. Tagliorette. 2001. Demanddriven commercial whalewatching in Península Valdés (Patagonia): conservation implications for right whales. Journal of Cetacean Research and Management, Cambridge, 2: 145-151.

Santos, M.C.O.; S. Rosso; S. Siciliano; A.N. Zerbini; E. Zampirolli; A. Vicente \& F. Alvarenga. 2000. Behavioral observations of the marine tucuxi dolphin (Sotalia fluviatilis) in São Paulo estuarine waters, southeastern Brazil. Aquatic Mammals, Moline, 26 (3): 260-267.

Spinelli, L.H.; L.F. Nascimento \& M.E. Yamamoto. 2002. Identificação e descrição da brincadeira em uma espécie pouco estudada, o boto cinza (Sotalia fluviatilis), em seu ambiente natural. Estudos em Psicologia, Natal, 7 (1): 165-171.

WATKINS, W.A. 1986. Whale reactions to human activities in Cape Cod waters. Marine Mammal Science, Lawrence, 2 (4): 251-262.

Williams, R.; A.W. Trites \& D.E. BAin. 2002. Behavioural responses of killer whales (Orcinus orca) to whale-watching boats: opportunistic observations and experimental approaches. Journal of Zoology, Cambridge, 256: 255-270. 